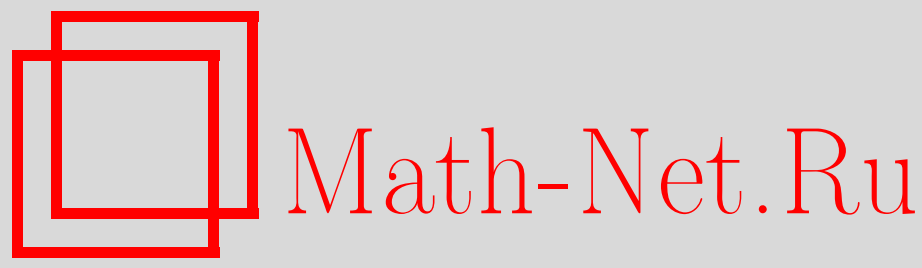

Т. В. Швец, В. Т. Швец, Вариационный подход к исследованию электрического сопротивления простых неупорядоченных металлов, ТМФ, 2011, том 166, номер 2, 282298

DOI: https://doi.org/10.4213/tmf6610

Использование Общероссийского математического портала Math-Net.Ru подразумевает, что вы прочитали и согласны с пользовательским соглашением http://www.mathnet.ru/rus/agreement

Параметры загрузки:

IP : 18.234 .156 .22

26 апреля 2023 г., 08:13:41

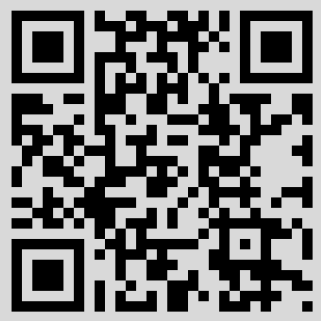




\title{
ВАРИАЦИОННЫЙ ПОДХОД К ИССЛЕДОВАНИЮ ЭЛЕКТРИЧЕСКОГО СОПРОТИВЛЕНИЯ ПРОСТЫХ НЕУПОРЯДОЧЕННЫХ МЕТАЛЛОВ
}

\begin{abstract}
На основе вариационного принципа получено выражение для коэффициента электрического сопротивления простых неупорядоченных металлов, справедливое в четвертом порядке теории возмущений по электрон-ионному взаимодействию. Ионная подсистема считалась статической, температурные поправки не учитывались. Параметры расцепления функций Грина старших порядков, возникающие при получении квантового кинетического уравнения, выбирались из условия совпадения уравнения Больцмана и квантового кинетического уравнения в низшем порядке теории возмущений. Вычисление электрического сопротивления неупорядоченного металла сводилось к поиску минимума соответствующего функционала. Такой поход позволил впервые вычислить вклад перекрестного рассеяния в электрическое сопротивление неупорядоченных металлов в предельном случае низких температур. Во втором и третьем порядках теории возмущений воспроизведены известные результаты. Показано, что в четвертом и более высоких порядках коэффициент электрического сопротивления выражается не только через время релаксации, но и через плотность состояний взаимодействующего с ионами электронного газа.
\end{abstract}

Ключевые слова: вариационный метод, метод функций Грина, электрическое сопротивление, теория возмущений, квантовое кинетическое уравнение.

\section{1. ВВЕДЕНИЕ}

Наличие малого параметра, которым является псевдопотенциал электрон-ионного взаимодействия, позволяет вычислять различные характеристики простых неупорядоченных металлов в рамках теории возмущений. В каждом конкретном случае возникает свой ряд теории возмущений. Для коэффициента удельного электрического сопротивления простых жидких металлов первый член ряда, квадратичный по псевдопотенциалу, был получен еще в работе [1] (см. также библиографию в книгах [2], [3]). Это так называемая формула Займана. Оказалось, что наиболее важным фактором, влияющим на результаты численных расчетов, является учет высших порядков теории возмущений. При этом теория возмущений строилась не

* Одесская государственная академия холода, Одесса, Украина.

E-mail: tarval@breezein.net 
для коэффициента удельного сопротивления, а для обратного времени релаксации, характеризующего данный процесс [4]-[12]. В то же время, как показывает теоретический анализ, приближение времени релаксации справедливо для электрического сопротивления при учете членов ряда теории возмущений не выше третьего порядка. Но даже в третьем порядке теории возмущений цитированные выше работы не были успешными. Их аналитические результаты не согласовывались друг с другом, а результаты численных расчетов были немногочисленными и зачастую бессмысленными. Существенный прогресс в построении теории возмущения достигнут в более поздних работах [13]-[19], где в приближении времени релаксации член третьего порядка по псевдопотенциалу был получен и численно рассчитан практически для всех простых жидких металлов. Оказалось, что выход за рамки приближения времени релаксации требует аккуратного учета эффектов четвертого порядка. Отметим, что эффекты четвертого порядка даже для простых металлов могут быть весьма существенными. Они имеют порядок величины $\hbar / \varepsilon_{\mathrm{F}} \tau$, где $\varepsilon_{\mathrm{F}}-$ энергия Ферми, a $\tau$ - время релаксации. Этот безразмерный параметр, характеризующий затухание состояний свободных электронов за счет взаимодействия, для неупорядоченных простых металлов имеет значение от нескольких процентов до нескольких десятков процентов. Наиболее кардинально эти эффекты могут влиять на соотношения между различными кинетическими коэффициентами или компонентами тензора одного из них. Особенно актуальным данный вопрос становится в случае необходимости приближенного суммирования всего ряда теории возмущений, например, в случае вычисления электрического сопротивления металлического водорода вблизи точки перехода металл-диэлектрик [20]-[22]. Одним из абсолютно не исследованных эффектов четвертого порядка является перекрестное рассеяние электронов проводимости внешним полем и полем ионов. Далее идут учет перенормировки спектра свободных электронов, отличие плотности состояний и функции распределения взаимодействующего электронного газа от плотности состояний и функции распределения свободных электронов. Разумеется, важен и вклад четвертого порядка в обратное время релаксации. Данная работа посвящена исследованию всего комплекса вышеперечисленных вопросов.

\section{2. КОЭФФИЦИЕНТ ЭЛЕКТРИЧЕСКОГО СОПРОТИВЛЕНИЯ}

Гамильтониан электронной подсистемы простого металла будем рассматривать в соответствии с дифракционной моделью металла, где электрон-электронное взаимодействие учитывается посредством экранирования электрон-ионного взаимодействия [3]:

$$
H(t)=\sum_{\mathbf{k}}\left[\varepsilon_{k}-\mu\right] a_{\mathbf{k}}^{+}(t) a_{\mathbf{k}}(t)+V^{-1} \sum_{\mathbf{k}, \mathbf{q}} w(q) \rho(\mathbf{q}) a_{\mathbf{k}}^{+}(t) a_{\mathbf{k}+\mathbf{q}}(t) .
$$

Здесь $a_{\mathbf{k}}^{+}, a_{\mathbf{k}}$ - операторы рождения и уничтожения электронов в состоянии с волновым вектором $\mathbf{k}, \varepsilon_{k}=\hbar^{2} k^{2} /(2 m)$ - энергия свободного электронного газа, $\mu$ - химический потенциал электронной подсистемы, $w(q)$ - формфактор локального модельного псевдопотенциала электрон-ионного взаимодействия, $\rho(\mathbf{q})$ - фурье-образ плотности ионов, $V$ - объем системы. Ионную подсистему будем считать статической из-за малости вклада динамики ионной подсистемы в кинетические коэффициенты неупорядоченных металлов [3]. 
Для нахождения коэффициента электропроводности будем использовать теорию линейной реакции Кубо, согласно которой в постоянном и однородном электрическом поле для изотропного металла он имеет вид [3]

$$
\sigma=-\frac{\hbar}{3 V k_{\mathrm{B}} T} \operatorname{Im}\left\langle\left\langle\mathbf{I}(t) \mathbf{I}\left(t^{\prime}\right)\right\rangle\right\rangle_{0} .
$$

Здесь оператор электрического тока

$$
\mathbf{I}(t)=\frac{e \hbar}{m} \sum_{\mathbf{k}} \mathbf{k} a_{\mathbf{k}}^{+}(t) a_{\mathbf{k}}(t),
$$

двойные угловые скобки обозначают двухвременну́ю запаздывающую функцию Грина, нижний индекс - нулевую компоненту Фурье.

Функцию Грина, определяющую коэффициент электропроводности, будем искать методом использования уравнения движения [23]. Первое уравнение имеет вид

$$
\begin{aligned}
& \hbar \omega\left\langle\left\langle a_{\mathbf{k}}^{+}(t) a_{\mathbf{k}}(t) \mathbf{I}(0)\right\rangle\right\rangle_{\omega}=\left\langle a_{\mathbf{k}}^{+}(0) a_{\mathbf{k}}(0) \mathbf{I}(0)\right\rangle+ \\
& \quad+V^{-1} \sum_{\mathbf{q}}^{\prime}\left\{w(\mathbf{q})\left\langle\left\langle a_{\mathbf{k}}^{+}(t) \rho(-\mathbf{q}, t) a_{\mathbf{k}+\mathbf{q}}(t) \mathbf{I}(0)\right\rangle\right\rangle_{\omega}-\right. \\
& \left.\quad-\left\langle\left\langle a_{\mathbf{k}+\mathbf{q}}^{+}(t) \rho(\mathbf{q}, t) a_{\mathbf{k}}(t) \mathbf{I}(0)\right\rangle\right\rangle_{\omega}\right\} .
\end{aligned}
$$

Для функций Грина, входящих в правую часть этого уравнения, в свою очередь составим уравнения движения типа

$$
\begin{aligned}
\left(\varepsilon_{\mathbf{k}}-\right. & \left.\varepsilon_{\mathbf{k}+\mathbf{q}}+\hbar \omega\right)\left\langle\left\langle a_{\mathbf{k}}^{+}(t) \rho^{i}(-\mathbf{q}) a_{\mathbf{k}+\mathbf{q}}(t) \mathbf{I}(0)\right\rangle\right\rangle_{\omega}=\left\langle a_{\mathbf{k}}^{+}(0) \rho^{i}(-\mathbf{q}) a_{\mathbf{k}+\mathbf{q}}(0) \mathbf{I}(0)\right\rangle+ \\
& +V^{-1} w(q)\left\{\left\langle\left\langle a_{\mathbf{k}}^{+}(t) \rho(-\mathbf{q}) \rho(\mathbf{q}) a_{\mathbf{k}}(t) \mathbf{I}(0)\right\rangle\right\rangle_{\omega}-\right. \\
& \left.-\left\langle\left\langle a_{\mathbf{k}+\mathbf{q}}^{+}(t) \rho(\mathbf{q}) \rho(-\mathbf{q}) a_{\mathbf{k}+\mathbf{q}}(t) \mathbf{I}(0)\right\rangle\right\rangle_{\omega}\right\}+ \\
& +V^{-1} \sum_{\mathbf{q}^{\prime}}^{\prime}\left\{w\left(\mathbf{q}^{\prime}\right)\left\langle\left\langle a_{\mathbf{k}}^{+}(t) \rho(-\mathbf{q}) \rho\left(-\mathbf{q}^{\prime}\right) a_{\mathbf{k}+\mathbf{q}+\mathbf{q}^{\prime}}(t) \mathbf{I}(0)\right\rangle\right\rangle_{\omega}-\right. \\
& \left.-\left\langle\left\langle a_{\mathbf{k}+\mathbf{q}^{\prime}}^{+}(t) \rho(\mathbf{q}) \rho\left(-\mathbf{q}^{\prime}\right) a_{\mathbf{k}+\mathbf{q}}(t) \mathbf{I}(0)\right\rangle\right\rangle_{\omega}\right\} .
\end{aligned}
$$

Правая часть уравнения (5) содержит два вида функций Грина. Структура одних определяется слабо связанными, других - сильно связанными средними. Для функций Грина второго вида вновь следует составить уравнения движения, продолжая эту цепочку уравнений, содержащую только функции Грина первого вида, до бесконечности. Для ее замыкания относительно искомой функции Грина следует выполнить расцепление типа

$$
\left\langle\left\langle a_{\mathbf{k}}^{+}(t) \rho(-\mathbf{q}, t) \rho(\mathbf{q}) a_{\mathbf{k}}(t) \mathbf{I}(0)\right\rangle\right\rangle_{\omega}=f(\mathbf{k}, \mathbf{k}+\mathbf{q})\langle\rho(-\mathbf{q}) \rho(\mathbf{q})\rangle\left\langle\left\langle a_{\mathbf{k}}^{+}(t) a_{\mathbf{k}}(t) \mathbf{I}(0)\right\rangle\right\rangle_{\omega} .
$$

Здесь функция $f(\mathbf{k}, \mathbf{k}+\mathbf{q})$ подбирается из условий выполнения каких-либо важных свойств симметрии всего уравнения. Этот выбор мы конкретизируем ниже. Объединяя уравнения цепочки, получим квантовое кинетическое уравнение

$$
R(\mathbf{k})+\sum_{\mathbf{k}^{\prime}} T\left\{\left\langle\left\langle a_{\mathbf{k}}^{+}(t) a_{\mathbf{k}}(t) \mathbf{I}(0)\right\rangle\right\rangle_{0}-\left\langle\left\langle a_{\mathbf{k}^{\prime}}^{+}(t) a_{\mathbf{k}^{\prime}}(t) \mathbf{I}(0)\right\rangle\right\rangle_{0}\right\}=0,
$$


где свободный член уравнения

$$
\begin{aligned}
R(\mathbf{k})= & \left\langle a_{\mathbf{k}}^{+}(0) a_{\mathbf{k}}(0) \mathbf{I}(0)\right\rangle+V^{-1} \sum_{\mathbf{k}^{\prime}} w\left(\left|\mathbf{k}^{\prime}-\mathbf{k}\right|\right)\left[\frac{\left\langle a_{\mathbf{k}}^{+}(0) \rho\left(\mathbf{k}^{\prime}-\mathbf{k}\right) a_{\mathbf{k}^{\prime}}(0) \mathbf{I}(0)\right\rangle}{\varepsilon_{\mathbf{k}}-\varepsilon_{\mathbf{k}^{\prime}}+i \delta}-\right. \\
& \left.-\frac{\left\langle a_{\mathbf{k}^{\prime}}^{+}(0) \rho\left(\mathbf{k}-\mathbf{k}^{\prime}\right) a_{\mathbf{k}}(0) \mathbf{I}(0)\right\rangle}{\varepsilon_{\mathbf{k}^{\prime}}-\varepsilon_{\mathbf{k}}+i \delta}\right]+\cdots
\end{aligned}
$$

описывает рассеяние электронов внешним электрическим полем, если рассчитывать его в нулевом порядке по псевдопотенциалу, и перекрестное рассеяние внешним полем и полем ионов, если учитывать члены более высокого порядка по псевдопотенциалу.

Пользуясь терминологией уравнения Больцмана, эти члены уравнения следует назвать полевым и перекрестным членами соответственно [24]. Интегральный член уравнения

$$
\begin{gathered}
T\left\{\left\langle\left\langle a_{\mathbf{k}}^{+}(t) a_{\mathbf{k}}(t) \mathbf{I}(0)\right\rangle\right\rangle_{0}-\left\langle\left\langle a_{\mathbf{k}^{\prime}}^{+}(t) a_{\mathbf{k}^{\prime}}(t) \mathbf{I}(0)\right\rangle\right\rangle_{0}\right\}=\frac{N}{V} w^{2}\left(\left|\mathbf{k}^{\prime}-\mathbf{k}\right|\right) S\left(\left|\mathbf{k}^{\prime}-\mathbf{k}\right|\right) \times \\
\quad \times\left\{\frac{f\left(\mathbf{k}, \mathbf{k}^{\prime}\right)\left\langle\left\langle a_{\mathbf{k}}^{+}(t) a_{\mathbf{k}}(t) \mathbf{I}(0)\right\rangle\right\rangle_{0}}{\varepsilon_{\mathbf{k}}-\varepsilon_{\mathbf{k}^{\prime}}+i \delta}-\frac{f\left(\mathbf{k}^{\prime}, \mathbf{k}\right)\left\langle\left\langle a_{\mathbf{k}^{\prime}}^{+}(t) a_{\mathbf{k}^{\prime}}(t) \mathbf{I}(0)\right\rangle\right\rangle_{0}}{\varepsilon_{\mathbf{k}^{\prime}}-\varepsilon_{\mathbf{k}}+i \delta}\right\}+\cdots
\end{gathered}
$$

описывает рассеяние электронов проводимости только полем ионов - столкновительный член в терминах уравнения Больцмана. Многоточие обозначает члены более высокого порядка по псевдопотенциалу, $S(q)$ - двухчастичный структурный фактор ионной подсистемы. Далее, нам понадобится это уравнение в низшем порядке теории возмущений. Тогда его свободный член следует взять в нулевом порядке по псевдопотенциалу. В этом случае

$$
\left\langle a_{\mathbf{k}}^{+}(0) a_{\mathbf{k}}(0) \mathbf{I}(0)\right\rangle_{0}=\frac{e \hbar}{m} \sum_{\mathbf{k}^{\prime}} \mathbf{k}^{\prime}\left\langle a_{\mathbf{k}}^{+}(0) a_{\mathbf{k}}(0) a_{\mathbf{k}^{\prime}}^{+}(0) a_{\mathbf{k}^{\prime}}(0)\right\rangle_{0} .
$$

Используя теорему Вика-Блоха-Доминисиса для невзаимодействующих электронов [25] и выписывая только члены, дающие ненулевой вклад в сумму, получим

$$
\left\langle a_{\mathbf{k}}^{+}(0) a_{\mathbf{k}}(0) a_{\mathbf{k}^{\prime}}^{+}(0) a_{\mathbf{k}^{\prime}}(0)\right\rangle_{0}=n_{0}(k)\left[1-n_{0}(k)\right] \Delta\left(\mathbf{k}-\mathbf{k}^{\prime}\right),
$$

где $n_{0}(k)$ - функция распределения Ферми-Дирака. Таким образом, используя формулу Сохоцкого, в низшем порядке теории возмущений получим квантовое кинетическое уравнение в виде

$$
\begin{gathered}
\frac{e \hbar}{m} \mathbf{k} n_{0}(k)\left[1-n_{0}(k)\right]=i \frac{2 \pi N}{V^{2}} \sum_{\mathbf{k}^{\prime}} \frac{w^{2}\left(\left|\mathbf{k}-\mathbf{k}^{\prime}\right|\right)}{\varepsilon^{2}\left(\left|\mathbf{k}-\mathbf{k}^{\prime}\right|\right)} S\left(\left|\mathbf{k}-\mathbf{k}^{\prime}\right|\right) \delta\left(\varepsilon_{\mathbf{k}}-\varepsilon_{\mathbf{k}^{\prime}}\right) \times \\
\times\left\{f\left(k, k^{\prime}\right)\left\langle\left\langle a_{\mathbf{k}}^{+}(t) a_{\mathbf{k}}(t) \mathbf{I}(0)\right\rangle\right\rangle_{0}-f\left(k^{\prime}, k\right)\left\langle\left\langle a_{\mathbf{k}^{\prime}}^{+}(t) a_{\mathbf{k}^{\prime}}(t) \mathbf{I}(0)\right\rangle\right\rangle_{0}\right\} .
\end{gathered}
$$

\section{3. УРАВНЕНИЕ БОЛЬЦМАНА}

Кинетическое уравнение Больцмана доказало свою высокую эффективность при описании электронных явлений переноса в металлах в первом неисчезающем порядке теории возмущений. Оно получено на основании совершенно ясных физических 
соображений и не содержит неконтролируемых приближений. Полученное нами квантовое кинетическое уравнение такое приближение содержит. Им является расцепление функций Грина старшего порядка. Минимизировать неопределенность, возникающую при расцеплении, можно, сопоставляя квантовое кинетическое уравнение в низшем порядке теории возмущений и кинетическое уравнение Больцмана. Последнее в постоянном электрическом поле и в отсутствие градиента температур и магнитного поля можно записать в виде [26]

$$
-\frac{e}{\hbar} \frac{\partial \tilde{n}(\mathbf{k})}{\partial \mathbf{k}} \mathbf{E}=\sum_{\mathbf{k}^{\prime}}\left[\frac{\tilde{n}\left(\mathbf{k}^{\prime}\right)[1-\tilde{n}(\mathbf{k})]}{n_{0}\left(\mathbf{k}^{\prime}\right)\left[1-n_{0}(\mathbf{k})\right]}-\frac{\tilde{n}(\mathbf{k})\left[1-\tilde{n}\left(\mathbf{k}^{\prime}\right)\right]}{n_{0}(\mathbf{k})\left[1-n_{0}\left(\mathbf{k}^{\prime}\right)\right]}\right] n_{0}\left(\mathbf{k}^{\prime}\right)\left[1-n_{0}(\mathbf{k})\right] L_{2}\left(\mathbf{k}^{\prime}, \mathbf{k}\right) .
$$

Здесь $\tilde{n}(\mathbf{k})$ - функция распределения электронов проводимости в присутствии внешнего электрического поля и поля ионов. Вероятность перехода электрона при рассеянии на ионах из состояния с волновым вектором $\mathbf{k}$ в состояние с волновым вектором $\mathbf{k}^{\prime}$ в низшем порядке теории возмущений описывается функцией $L_{2}\left(\mathbf{k}, \mathbf{k}^{\prime}\right)$. В отсутствие внешних воздействий на электроны вероятности прямых и обратных переходов должны быть равны (принцип детального равновесия). При этом начальное состояние должно быть занято, а конечное - свободно, т. е.

$$
n_{0}(\mathbf{k})\left[1-n_{0}\left(\mathbf{k}^{\prime}\right)\right] L\left(\mathbf{k}, \mathbf{k}^{\prime}\right)=n_{0}\left(\mathbf{k}^{\prime}\right)\left[1-n_{0}(\mathbf{k})\right] L\left(\mathbf{k}^{\prime}, \mathbf{k}\right) .
$$

Мы привели уравнение Больцмана в виде, который автоматически удовлетворяет этому условию. Для металлов функция $\tilde{n}(\mathbf{r}, \mathbf{k})$ мало отличается от равновесной функции Ферми-Дирака. Кинетическое уравнение линеаризуется по отклонению функции $\tilde{n}(\mathbf{r})$ от ее равновесного значения следующим образом:

$$
\tilde{n}(\mathbf{k})=n_{0}(k)-\Phi(\mathbf{k}) \frac{n_{0}(k)\left[1-n_{0}(k)\right]}{k_{\mathrm{B}} T},
$$

где $\Phi(\mathbf{k})$ - новая неизвестная функция. После подстановки последнего выражения в уравнение Больцмана и его линеаризации имеем

$$
\frac{e \hbar}{m k_{\mathrm{B}} T} \mathbf{k} n_{0}(k)\left[1-n_{0}(k)\right] \mathbf{E}=\frac{1}{k_{\mathrm{B}} T} \sum_{\mathbf{k}^{\prime}}\left[\Phi(\mathbf{k})-\Phi\left(\mathbf{k}^{\prime}\right)\right] n_{0}\left(k^{\prime}\right)\left[1-n_{0}(k)\right] L_{2}\left(\mathbf{k}^{\prime}, \mathbf{k}\right) .
$$

Для сопоставления уравнения Больцмана и квантового кинетического уравнения необходимо входящую в последнее уравнение функцию Грина выразить через функцию $\Phi(\mathbf{k})$. Для этого сравним выражения для электрического тока, полученные с помощью этих функций. В первом случае

$$
I=\frac{e \hbar}{m k_{\mathrm{B}} T} \sum_{\mathbf{k}} \mathbf{k} n_{0}(k)\left[1-n_{0}(k)\right] \Phi(\mathbf{k})
$$

во втором случае

$$
I=\sigma E=-\frac{\hbar}{3} \frac{e \hbar}{m k_{\mathrm{B}} T V} \sum_{\mathbf{k}} \mathbf{k} \operatorname{Im}\left\langle\left\langle a_{\mathbf{k}}^{+}(t) a_{\mathbf{k}}(t) \mathbf{I}(0)\right\rangle\right\rangle_{0} E .
$$

Таким образом, искомая связь будет иметь вид

$$
-i \frac{\hbar}{3 V}\left\langle\left\langle a_{\mathbf{k}}^{+}(t) a_{\mathbf{k}}(t) \mathbf{I}(0)\right\rangle\right\rangle_{0} E=n_{0}(k)\left[1-n_{0}(k)\right] \Phi(\mathbf{k}) .
$$


Теперь квантовое кинетическое уравнение можно записать аналогично уравнению Больцмана:

$$
\begin{aligned}
& \frac{e \hbar}{m k_{\mathrm{B}} T} \mathbf{k} n_{0}(k)\left[1-n_{0}(k)\right] E=\frac{1}{k_{\mathrm{B}} T} \sum_{\mathbf{k}^{\prime}}\left\{f\left(k, k^{\prime}\right) n_{0}(k)\left[1-n_{0}(k)\right] \Phi(\mathbf{k}) L\left(\mathbf{k}, \mathbf{k}^{\prime}\right)-\right. \\
& \left.\quad-f\left(k^{\prime}, k\right) n_{0}\left(k^{\prime}\right)\left[1-n_{0}\left(k^{\prime}\right)\right] \Phi\left(\mathbf{k}^{\prime}\right) L_{2}\left(\mathbf{k}^{\prime}, \mathbf{k}\right)\right\}
\end{aligned}
$$

где для вероятности перехода между состояниями получено выражение

$$
L_{2}\left(\mathbf{k}^{\prime}, \mathbf{k}\right)=3 \frac{2 \pi N}{V \hbar} \frac{w^{2}\left(\left|\mathbf{k}-\mathbf{k}^{\prime}\right|\right)}{\varepsilon^{2}\left(\left|\mathbf{k}-\mathbf{k}^{\prime}\right|\right)} S\left(\left|\mathbf{k}-\mathbf{k}^{\prime}\right|\right) \delta\left(\varepsilon_{\mathbf{k}}-\varepsilon_{\mathbf{k}^{\prime}}\right)
$$

Подгоночные функции, возникшие при расцеплении функций Грина старшего порядка, теперь можно найти из условия совпадения квантового кинетического уравнения и уравнения Больцмана в низшем порядке теории возмущений. Очевидно, чTO

$$
f\left(k, k^{\prime}\right)=\frac{n_{0}\left(k^{\prime}\right)\left[1-n_{0}(k)\right]}{n_{0}(k)\left[1-n_{0}(k)\right]} .
$$

Эти функции учитывают асимметрию состояний, в которых осуществляется проводимость, и состояний, из которых забираются электроны, участвующие далее в переносе электрического заряда. В случае упругого рассеяния $k=k^{\prime}$ и $f\left(k, k^{\prime}\right)=$ $f\left(k^{\prime}, k\right)=1$. Именно подобие рассматриваемых уравнений позволяет интерпретировать каждый член квантового уравнения в терминах уравнения Больцмана. При учете членов высших порядков теории возмущений в последнем соотношении должна фигурировать функция распределения взаимодействующего электронного газа.

\section{4. ВАРИАЦИОННЫЙ ПРИНЦИП}

Наряду с квантовым кинетическим уравнением можно рассмотреть и функционал, вариацией которого оно может быть получено, аналогично тому, как это делается для уравнения Больцмана [26]:

$$
(\Phi, \hat{L} \Phi)=(\Phi, X)
$$

где

$$
\begin{aligned}
(\Phi, X) & =\sum_{\mathbf{k}} \Phi(\mathbf{k}) R(\mathbf{k}) E \\
(\Phi, \hat{L} \Phi) & =-\frac{1}{2} \sum_{\mathbf{k}, \mathbf{k}^{\prime}}\left[\Phi(\mathbf{k})-\Phi\left(\mathbf{k}^{\prime}\right)\right] T\left[\Phi(\mathbf{k})-\Phi\left(\mathbf{k}^{\prime}\right)\right]
\end{aligned}
$$

В этом разделе мы для простоты ограничимся низшим порядком теории возмущений, поскольку важна только симметрия этого уравнения, которая не зависит от порядка теории возмущений. В этом случае уравнение имеет вид

$$
\frac{e \hbar}{m} \sum_{\mathbf{k}} \Phi(\mathbf{k}) \mathbf{k} n_{0}(\mathbf{k})\left[1-n_{0}(\mathbf{k})\right] E=\frac{1}{2} \sum_{\mathbf{k}, \mathbf{k}^{\prime}}\left[\Phi(\mathbf{k})-\Phi\left(\mathbf{k}^{\prime}\right)\right]^{2} n_{0}\left(\mathbf{k}^{\prime}\right)\left[1-n_{0}(\mathbf{k})\right] L_{2}\left(\mathbf{k}^{\prime}, \mathbf{k}\right)
$$


Можно показать, что ядро функционала в правой части последнего уравнения эрмитово. В этом случае для квантового кинетического уравнения по аналогии с уравнением Больцмана можно сформулировать вариационный принцип, заключающийся в том [26], что решением квантового кинетического уравнения является функция $\Phi(\mathbf{k})$, обеспечивающая минимум функционала $(\Phi \hat{L} \Phi) /(\Phi, X)^{2}$.

Вычисляя скорость генерации энтропии в цепи с удельным сопротивлением $\rho$, по которой течет ток $I$, с помощью уравнения Больцмана для коэффициента электрического сопротивления было получено выражение [26]

$$
\rho=\frac{k_{\mathrm{B}} T(\Phi, \hat{L} \Phi) E^{2}}{(\Phi, X)^{2}} .
$$

Поскольку структура нашего квантового кинетического уравнения тождественна структуре уравнения Больцмана, эта формула будет справедлива и при использовании квантового кинетического уравнения.

\section{5. ВТОРОЙ И ТРЕТИЙ ПОРЯДКИ ТЕОРИИ ВОЗМУЩЕНИЙ}

Рассмотрим случай упругого рассеяния. Тогда решение кинетического уравнения, записанного во втором порядке теории возмущений по взаимодействию, известно. С точностью до постоянного сомножителя оно равно

$$
\Phi(\mathbf{k})=\mathbf{k} E
$$

В этом случае

$$
\frac{1}{k_{\mathrm{B}} T}(\Phi, X)=\frac{e V k_{\mathrm{F}}^{3}}{\pi^{2} \hbar} E .
$$

Соответственно

$$
(\Phi, \hat{L} \Phi)=\frac{3 V^{2} m k_{\mathrm{F}}^{3}}{\pi^{2} \hbar^{2}} \frac{1}{\tau_{2}\left(k_{\mathrm{F}}\right)},
$$

где обратное время релаксации

$$
\frac{1}{\tau_{2}\left(k_{\mathrm{F}}\right)}=\frac{m n}{4 \pi \hbar^{3} k_{\mathrm{F}}^{3}} \int_{0}^{2 k_{\mathrm{F}}} w^{2}(q) S(q) q^{3} d q .
$$

Тогда выражение для коэффициента удельного сопротивления имеет вид

$$
\rho=\frac{m}{e^{2} n} \frac{1}{\tau_{2}\left(k_{\mathrm{F}}\right)} .
$$

Преимущество вариационного метода проявляется тогда, когда точное решение кинетического уравнения найти невозможно.

Рассмотрим теперь член третьего порядка теории возмущений. В правую часть первого уравнения цепочки входят две функции Грина более высокого порядка по отношению к искомой, содержащие сильносвязанные средние. Каждое из новых 
двух уравнений движения содержит еще по две функции Грина более высокого порядка, подлежащие определению. Таким образом, на каждом шаге построения цепочки уравнений у нас удваивается количество функций Грина, подлежащих определению. На третьем шаге необходимо выписать уже восемь уравнений типа

$$
\begin{aligned}
&\left(\varepsilon_{\mathbf{k}}-\varepsilon_{\mathbf{k}+\mathbf{q}^{\prime}}+\mathbf{q}^{\prime \prime}+\hbar \omega\right)\left\langle\left\langle W\left(-\mathbf{q}^{\prime}\right) W\left(-\mathbf{q}^{\prime \prime}\right) a_{\mathbf{k}}^{+}(t) a_{\mathbf{k}+\mathbf{q}^{\prime}+\mathbf{q}^{\prime \prime}}(t) \mathbf{I}_{z}(0)\right\rangle\right\rangle_{\omega}= \\
&=\langle W\left(-\mathbf{q}^{\prime}\right) W\left(-\mathbf{q}^{\prime \prime}\right) a_{\mathbf{k}}^{+}(0) a_{\left.\mathbf{k}+\mathbf{q}^{\prime}+\mathbf{q}^{\prime \prime}(0) \mathbf{I}_{z}(0)\right\rangle+} \\
&+\left\langle\left\langle W\left(-\mathbf{q}^{\prime}\right) W\left(-\mathbf{q}^{\prime \prime}\right) W\left(\mathbf{q}^{\prime}+\mathbf{q}^{\prime \prime}\right) a_{\mathbf{k}}^{+}(t) a_{\mathbf{k}}(t) I_{z}(0)\right\rangle\right\rangle_{\omega}- \\
&-\left\langle\left\langle W\left(-\mathbf{q}^{\prime}\right) W\left(-\mathbf{q}^{\prime \prime}\right) W\left(\mathbf{q}^{\prime}+\mathbf{q}^{\prime \prime}\right) a_{\mathbf{k}+\mathbf{q}^{\prime}+\mathbf{q}^{\prime \prime}}(t) a_{\mathbf{k}+\mathbf{q}^{\prime}+\mathbf{q}^{\prime \prime}}(t) I_{z}(0)\right\rangle\right\rangle_{\omega}+ \\
&+\left\langle\left\langle W\left(-\mathbf{q}^{\prime}\right) W\left(-\mathbf{q}^{\prime \prime}\right) W\left(\mathbf{q}^{\prime \prime}\right) a_{\mathbf{k}}^{+}(t) a_{\mathbf{k}+\mathbf{q}^{\prime}}(t) I_{z}(0)\right\rangle\right\rangle_{\omega}- \\
&-\left\langle\left\langle W\left(-\mathbf{q}^{\prime}\right) W\left(-\mathbf{q}^{\prime \prime}\right) W\left(\mathbf{q}^{\prime \prime}\right) a_{\mathbf{k}+\mathbf{q}^{\prime \prime}}^{+}(t) a_{\mathbf{k}+\mathbf{q}^{\prime}}(t) I_{z}(0)\right\rangle\right\rangle_{\omega}+ \\
&+\left\langle\left\langle W\left(-\mathbf{q}^{\prime}\right) W\left(-\mathbf{q}^{\prime \prime}\right) W\left(\mathbf{q}^{\prime}\right) a_{\mathbf{k}}^{+}(t) a_{\mathbf{k}+\mathbf{q}^{\prime \prime}}(t) I_{z}(0)\right\rangle\right\rangle_{\omega}- \\
&-\left\langle\left\langle W\left(-\mathbf{q}^{\prime}\right) W\left(-\mathbf{q}^{\prime \prime}\right) W\left(\mathbf{q}^{\prime}\right) a_{\mathbf{k}+\mathbf{q}^{\prime}}^{+}(t) a_{\mathbf{k}+\mathbf{q}^{\prime \prime}}(t) I_{z}(0)\right\rangle\right\rangle_{\omega}+ \\
&+\sum_{\mathbf{q}^{\prime \prime}}\left\{\left\langle\left\langle W\left(-\mathbf{q}^{\prime}\right) W\left(-\mathbf{q}^{\prime \prime}\right) W\left(-\mathbf{q}^{\prime \prime \prime}\right) a_{\mathbf{k}}^{+}(t) a_{\mathbf{k}+\mathbf{q}^{\prime}+\mathbf{q}^{\prime \prime}+\mathbf{q}^{\prime \prime \prime}}(t) I_{z}(0)\right\rangle\right\rangle_{\omega}-\right. \\
&\left.-\left\langle\left\langle W\left(\mathbf{q}^{\prime \prime \prime}\right) W\left(-\mathbf{q}^{\prime}\right) W\left(-\mathbf{q}^{\prime \prime}\right) a_{\mathbf{k}+\mathbf{q}^{\prime \prime}}(t) a_{\mathbf{k}+\mathbf{q}^{\prime}+\mathbf{q}^{\prime \prime}}(t) I_{z}(0)\right\rangle\right\rangle_{\omega}\right\},
\end{aligned}
$$

где для простоты мы обозначили $W(\mathbf{q})=V^{-1} w(q) \rho(\mathbf{q})$. Первый член в правой части последнего уравнения описывает вклад перекрестных процессов рассеяния электронов начиная с членов третьего порядка. Второй и третий члены описывают вклад в столкновительный член кинетического уравнения начиная с членов третьего порядка. Четвертый - перенормировку энергии свободных электронов за счет электрон-ионного взаимодействия в выражении для столкновительного члена, первоначально выписанного во втором порядке теории возмущений. Пятый, шестой и седьмой члены отвечают за перенормировку энергии свободных электронов в столкновительных членах, соответствующих четвертому и более высоким порядкам теории возмущений. С точки зрения формального разложения коэффициента электрического сопротивления в ряд теории возмущений первый член соответствует эффектам пятого порядка, второй и третий члены - эффекту третьего порядка, четвертый - четвертого порядка, пятый, шестой и седьмой - эффектам пятого и более высоких порядков. Ограничимся рассмотрением перенормировки энергии свободных электронов при учете эффектов не выше четвертого порядка. Заметим, что рассмотрение членов теории возмущений выше четвертого порядка уже не приводит к учету качественно новых эффектов по сравнению с учетом членов второго, третьего и четвертого порядков. Поэтому четвертым порядком теории возмущений в данной работе мы и ограничимся.

Выписанное уравнение позволяет получить столкновительный член с учетом члена третьего порядка, и такое квантовое кинетическое уравнение было получено в работах [3], [14], [15], [20]. Если записать соответствующий этому уравнению функционал, то он будет иметь вид

$$
\frac{e \hbar}{m k_{\mathrm{B}} T} \mathbf{k} n_{0}(\mathbf{k})\left[1-n_{0}(\mathbf{k})\right] E=\frac{1}{k_{\mathrm{B}} T} \sum_{\mathbf{k}^{\prime}}\left[\Phi(\mathbf{k})-\Phi\left(\mathbf{k}^{\prime}\right)\right]^{2} n_{0}\left(\mathbf{k}^{\prime}\right)\left[1-n_{0}(\mathbf{k})\right] L\left(\mathbf{k}^{\prime}, \mathbf{k}\right),
$$


где вероятность переходов между состояниями равна

$$
\begin{aligned}
L\left(\mathbf{k}^{\prime}, \mathbf{k}\right)= & L_{2}\left(\mathbf{k}^{\prime}, \mathbf{k}\right)+L_{3}\left(\mathbf{k}^{\prime}, \mathbf{k}\right) \\
L_{3}\left(\mathbf{k}^{\prime}, \mathbf{k}\right)= & 3 \frac{4 \pi N}{V \hbar^{3}} \sum_{\mathbf{k}^{\prime \prime}}^{\prime} \frac{w\left(\left|\mathbf{k}-\mathbf{k}^{\prime}\right|\right) w\left(\left|\mathbf{k}^{\prime}-\mathbf{k}^{\prime \prime}\right|\right) w\left(\left|\mathbf{k}^{\prime \prime}-\mathbf{k}\right|\right)}{\varepsilon\left(\left|\mathbf{k}-\mathbf{k}^{\prime}\right|\right) \varepsilon\left(\left|\mathbf{k}^{\prime}-\mathbf{k}^{\prime \prime}\right|\right) \varepsilon\left(\left|\mathbf{k}^{\prime \prime}-\mathbf{k}\right|\right)} \times \\
& \times S\left(\mathbf{k}-\mathbf{k}^{\prime}, \mathbf{k}^{\prime}-\mathbf{k}^{\prime \prime}, \mathbf{k}^{\prime \prime}-\mathbf{k}\right) \frac{\delta\left(\varepsilon_{\mathbf{k}}-\varepsilon_{\mathbf{k}^{\prime}}\right)}{\varepsilon_{\mathbf{k}}-\varepsilon_{\mathbf{k}^{\prime \prime}}}
\end{aligned}
$$

Используя для $\Phi(\mathbf{k})$ точное решение кинетического уравнения в низшем порядке теории возмущений, для полевого члена с учетом члена третьего порядка получим

$$
(\Phi, \hat{L} \Phi)=(\Phi, \hat{L} \Phi)_{2}+(\Phi, \hat{L} \Phi)_{3}+\cdots=\frac{1}{2} \sum_{\mathbf{k}, \mathbf{k}^{\prime}}\left(k^{2}+k^{\prime 2}-2 \mathbf{k k}^{\prime}\right) n_{0}\left(\mathbf{k}^{\prime}\right)\left[1-n_{0}(\mathbf{k})\right] L\left(\mathbf{k}^{\prime}, \mathbf{k}\right)
$$

Вклад второго порядка в столкновительный член аналогичен предыдущему. Вклад третьего порядка имеет вид

$$
\begin{gathered}
\frac{1}{k_{\mathrm{B}} T}(\Phi, \hat{L} \Phi)_{3}=\frac{3 V^{2} m k_{\mathrm{F}}^{3}}{\pi^{2} \hbar^{2}} \frac{1}{\tau_{3}\left(k_{\mathrm{F}}\right)}, \\
\frac{1}{\tau_{3}\left(k_{\mathrm{F}}\right)}=\frac{2 \pi n \pi^{2} \hbar^{2}}{V^{3} m \hbar^{3} k_{\mathrm{F}}^{3} k_{\mathrm{B}} T} \sum_{\mathbf{k}, \mathbf{k}^{\prime}, \mathbf{k}^{\prime \prime}}\left(k^{2}+k^{\prime 2}-2 \mathbf{k k}^{\prime}\right) n_{0}\left(\mathbf{k}^{\prime}\right)\left[1-n_{0}(\mathbf{k})\right] \times \\
\times \frac{w\left(\left|\mathbf{k}-\mathbf{k}^{\prime}\right|\right)}{\varepsilon\left(\left|\mathbf{k}-\mathbf{k}^{\prime}\right|\right)} \frac{w\left(\left|\mathbf{k}^{\prime}-\mathbf{k}^{\prime \prime}\right|\right)}{\varepsilon\left(\left|\mathbf{k}^{\prime}-\mathbf{k}^{\prime \prime}\right|\right)} \frac{w\left(\left|\mathbf{k}^{\prime \prime}-\mathbf{k}\right|\right)}{\varepsilon\left(\left|\mathbf{k}^{\prime \prime}-\mathbf{k}\right|\right)} S\left(\mathbf{k}-\mathbf{k}^{\prime}, \mathbf{k}^{\prime}-\mathbf{k}^{\prime \prime}, \mathbf{k}^{\prime \prime}-\mathbf{k}\right) \frac{\delta\left(\varepsilon_{\mathbf{k}}-\varepsilon_{\mathbf{k}^{\prime}}\right)}{\varepsilon_{\mathbf{k}}-\varepsilon_{\mathbf{k}^{\prime \prime}}},
\end{gathered}
$$

что фактически совпадает с выражением, полученным в других работах при приближенном решении кинетического уравнения [3], [14], [15], [20]. Это выражение также следует рассматривать как приближенное, поскольку в качестве пробной взята функция, являющаяся точным решением кинетического уравнения в низшем порядке теории возмущений, но не являющаяся таковой для кинетического уравнения, записанного в более высоких порядках теории возмущений. При использовании конволюционного приближения для трехчастичного структурного фактора кратность интегрирования может быть существенно понижена, и окончательное выражение совпадает с результатами предыдущих работ.

\section{6. ПЕРЕКРЕСТНЫЙ ЧЛЕН КВАНТОВОГО КИНЕТИЧЕСКОГО УРАВНЕНИЯ}

При рассмотрении членов четвертого и высших порядков теории возмущений недостаточно вычислить только обратное время релаксации с точностью до членов четвертого порядка. Необходимо учитывать и целый ряд более тонких эффектов. Одним из таких неисследованных эффектов четвертого порядка является перекрестное рассеяние электронов проводимости внешним электрическим полем и полем ионов. При этом в качестве пробной функции снова возьмем точное решение 
кинетического уравнения во втором порядке теории возмущений. Имеем

$$
\begin{gathered}
(\Phi, X)=\left(\Phi, X_{1}\right)+\left(\Phi, X_{2}\right), \\
\left(\Phi, X_{1}\right)=\sum_{\mathbf{k}} \mathbf{k}\left\langle a_{\mathbf{k}}^{+}(0) a_{\mathbf{k}}(0) \mathbf{I}(0)\right\rangle E \\
\left(\Phi, X_{2}\right)=\frac{1}{V} \sum_{\mathbf{k}, \mathbf{k}^{\prime}}^{\prime} \mathbf{k} w\left(\left|\mathbf{k}-\mathbf{k}^{\prime}\right|\right)\left[\frac{\left\langle a_{\mathbf{k}}^{+}(0) \rho^{i}\left(\mathbf{k}^{\prime}-\mathbf{k}\right) a_{\mathbf{k}^{\prime}}(0) \mathbf{I}(0)\right\rangle}{\varepsilon_{\mathbf{k}}-\varepsilon_{\mathbf{k}^{\prime}}+i \delta}-\right. \\
\left.-\frac{\left\langle a_{\mathbf{k}^{\prime}}^{+}(0) \rho^{i}\left(\mathbf{k}-\mathbf{k}^{\prime}\right) a_{\mathbf{k}}(0) \mathbf{I}(0)\right\rangle}{\varepsilon_{\mathbf{k}^{\prime}}-\varepsilon_{\mathbf{k}}+i \delta}\right] E .
\end{gathered}
$$

Используя формулу Сохоцкого и меняя местами индексы суммирования во втором слагаемом под знаком суммы, получаем

$$
\left(\Phi, X_{2}\right)_{2}=\frac{1}{V} \sum_{\mathbf{k}, \mathbf{k}^{\prime}}^{\prime}\left(\mathbf{k}-\mathbf{k}^{\prime}\right) w\left(\left|\mathbf{k}-\mathbf{k}^{\prime}\right|\right) \frac{\left\langle a_{\mathbf{k}}^{+}(0) \rho^{i}\left(\mathbf{k}-\mathbf{k}^{\prime}\right) a_{\mathbf{k}}(0) \mathbf{I}(0)\right\rangle}{\varepsilon_{\mathbf{k}}-\varepsilon_{\mathbf{k}^{\prime}}} E .
$$

В свою очередь уравнение движения для термодинамических средних имеет вид

$$
\begin{aligned}
\left(\varepsilon_{\mathbf{k}}-\right. & \left.\varepsilon_{\mathbf{k}+\mathbf{q}}\right)\left\langle a_{\mathbf{k}}^{+} \rho^{i}(-\mathbf{q}) a_{\mathbf{k}+\mathbf{q}} \mathbf{I}\right\rangle=\frac{e \hbar}{V m} \sum_{\mathbf{k}^{\prime}, \mathbf{q}^{\prime}} \mathbf{k}^{\prime} w\left(q^{\prime}\right) \times \\
& \times\left\{\left\langle a_{\mathbf{k}}^{+} \rho^{i}(-\mathbf{q}) \rho^{i}\left(-\mathbf{q}^{\prime}\right) a_{\mathbf{k}+\mathbf{q}+\mathbf{q}^{\prime}} a_{\mathbf{k}^{\prime}}^{+} a_{\mathbf{k}^{\prime}}\right\rangle-\left\langle a_{\mathbf{k}+\mathbf{q}^{\prime}}^{+} \rho^{i}(-\mathbf{q}) \rho^{i}\left(\mathbf{q}^{\prime}\right) a_{\mathbf{k}+\mathbf{q}} a_{\mathbf{k}^{\prime}}^{+} a_{\mathbf{k}^{\prime}}\right\rangle+\right. \\
& \left.+\left\langle a_{\mathbf{k}}^{+} \rho^{i}(-\mathbf{q}) \rho^{i}\left(-\mathbf{q}^{\prime}\right) a_{\mathbf{k}+\mathbf{q}} a_{\mathbf{k}^{\prime}}^{+} a_{\mathbf{k}^{\prime}+\mathbf{q}^{\prime}}\right\rangle-\left\langle a_{\mathbf{k}}^{+} \rho^{i}(-\mathbf{q}) \rho^{i}\left(\mathbf{q}^{\prime}\right) a_{\mathbf{k}+\mathbf{q}} a_{\mathbf{k}^{\prime}+\mathbf{q}^{\prime}}^{+} a_{\mathbf{k}^{\prime}}\right\rangle\right\} .
\end{aligned}
$$

Термодинамические средние в правой части этого уравнения достаточно рассчитать в нулевом порядке по псевдопотенциалу. Тогда

$$
\begin{aligned}
\left(\varepsilon_{\mathbf{k}}-\right. & \left.\varepsilon_{\mathbf{k}+\mathbf{q}}\right)\left\langle a_{\mathbf{k}}^{+} \rho^{i}(-\mathbf{q}) a_{\mathbf{k}+\mathbf{q}} \mathbf{I}\right\rangle=\frac{N}{V} \frac{e \hbar}{m} w(q) S(q) \sum_{\mathbf{k}^{\prime}} \mathbf{k}^{\prime} \times \\
& \times\left\{\left\langle a_{\mathbf{k}}^{+} a_{\mathbf{k}} a_{\mathbf{k}^{\prime}}^{+} a_{\mathbf{k}^{\prime}}\right\rangle-\left\langle a_{\mathbf{k}+\mathbf{q}}^{+} a_{\mathbf{k}+\mathbf{q}} a_{\mathbf{k}^{\prime}}^{+} a_{\mathbf{k}^{\prime}}\right\rangle+\left\langle a_{\mathbf{k}}^{+} a_{\mathbf{k}+\mathbf{q}} a_{\mathbf{k}^{\prime}}^{+} a_{\mathbf{k}^{\prime}-\mathbf{q}}\right\rangle-\left\langle a_{\mathbf{k}}^{+} a_{\mathbf{k}+\mathbf{q}} a_{\mathbf{k}^{\prime}+\mathbf{q}}^{+} a_{\mathbf{k}^{\prime}}\right\rangle\right\} .
\end{aligned}
$$

В нулевом порядке по электрон-ионному взаимодействию

$$
\left\langle a_{\mathbf{k}}^{+} a_{\mathbf{k}} a_{\mathbf{k}^{\prime}}^{+} a_{\mathbf{k}^{\prime}}\right\rangle=\left\langle a_{\mathbf{k}}^{+} a_{\mathbf{k}}\right\rangle\left[1-\left\langle a_{\mathbf{k}}^{+} a_{\mathbf{k}}\right\rangle\right] \Delta\left(\mathbf{k}-\mathbf{k}^{\prime}\right)+\left\langle a_{\mathbf{k}}^{+} a_{\mathbf{k}}\right\rangle\left\langle a_{\mathbf{k}^{\prime}}^{+} a_{\mathbf{k}^{\prime}}\right\rangle,
$$

где второе слагаемое в правой части не дает вклада в сумму по $\mathbf{k}^{\prime}$ из-за сферической симметрии задачи. Аналогично находятся и остальные средние. Таким образом,

$$
\begin{aligned}
& \left(\varepsilon_{\mathbf{k}}-\varepsilon_{\mathbf{k}+\mathbf{q}}\right)\left\langle a_{\mathbf{k}}^{+}(0) \rho^{i}(-\mathbf{q}) a_{\mathbf{k}+\mathbf{q}}(0) \mathbf{I}(0)\right\rangle=\frac{N}{V} \frac{e \hbar}{m} w(q) S(q) \times \\
& \quad \times\left\{\mathbf{k} n_{0}(\mathbf{k})\left[1-n_{0}(\mathbf{k})\right]-(\mathbf{k}+\mathbf{q}) n_{0}(\mathbf{k}+\mathbf{q})\left[1-n_{0}(\mathbf{k}+\mathbf{q})\right]+\mathbf{q} n_{0}(\mathbf{k})\left[1-n_{0}(\mathbf{k}+\mathbf{q})\right]\right\} .
\end{aligned}
$$

Теперь

$$
\begin{aligned}
\left(\Phi, X_{2}\right)= & E \frac{N}{V^{3}} \frac{e \hbar}{m} \sum_{\mathbf{k}, \mathbf{k}^{\prime}}^{\prime} w^{2}\left(\left|\mathbf{k}-\mathbf{k}^{\prime}\right|\right) S\left(\left|\mathbf{k}-\mathbf{k}^{\prime}\right|\right) \times \\
& \times\left\{2\left(k^{2}-\mathbf{k k}^{\prime}\right) \frac{n_{0}(\mathbf{k})\left[1-n_{0}(\mathbf{k})\right]}{\left(\varepsilon_{\mathbf{k}}-\varepsilon_{\mathbf{k}^{\prime}}\right)^{2}}-\left(\mathbf{k}-\mathbf{k}^{\prime}\right)^{2} \frac{n_{0}(\mathbf{k})\left[1-n_{0}\left(\mathbf{k}^{\prime}\right)\right]}{\left(\varepsilon_{\mathbf{k}}-\varepsilon_{\mathbf{k}^{\prime}}\right)^{2}}\right\} .
\end{aligned}
$$


Вычислим во втором порядке по электрон-ионному взаимодействию следующий член квантового кинетического уравнения:

$$
\left(\Phi, X_{1}\right)=\sum_{\mathbf{k}} \mathbf{k}\left\langle a_{\mathbf{k}}^{+}(0) a_{\mathbf{k}}(0) \mathbf{I}(0)\right\rangle E=\frac{e \hbar}{m} \sum_{\mathbf{k}, \mathbf{k}^{\prime}} \mathbf{k k}^{\prime}\left\langle a_{\mathbf{k}}^{+}(0) a_{\mathbf{k}}(0) a_{\mathbf{k}^{\prime}}^{+}(0) a_{\mathbf{k}^{\prime}}(0)\right\rangle E .
$$

Средние в правой части этого уравнения необходимо вычислить во втором порядке теории возмущений, представляя их суммой всех возможных парных произведений:

$$
\left\langle a_{\mathbf{k}}^{+}(0) a_{\mathbf{k}}(0) a_{\mathbf{k}^{\prime}}^{+}(0) a_{\mathbf{k}^{\prime}}(0)\right\rangle=\left\langle\left\langle a_{\mathbf{k}}^{+}(0) a_{\mathbf{k}^{\prime}}(0)\right\rangle\left\langle a_{\mathbf{k}^{\prime}}(0) a_{\mathbf{k}}^{+}(0)\right\rangle+\left\langle a_{\mathbf{k}}^{+}(0) a_{\mathbf{k}}(0)\right\rangle\left\langle a_{\mathbf{k}^{\prime}}^{+}(0) a_{\mathbf{k}^{\prime}}(0)\right\rangle\right\rangle .
$$

Здесь внутреннее усреднение выполняется по координатам электронной подсистемы, а внешнее - ионной. Второе слагаемое не дает вклада в соответствующую сумму из-за ее симметрии при суммировании по волновым векторам $\mathbf{k}$ и $\mathbf{k}^{\prime}$. Удобно выделить из оставшегося слагаемого диагональное и недиагональное слагаемые:

$$
\begin{aligned}
\left(\Phi, X_{1}\right) & =\left(\Phi, X_{1,1}\right)+\left(\Phi, X_{1,2}\right), \\
\left(\Phi, X_{1,1}\right) & =\frac{e \hbar}{m} \sum_{\mathbf{k}} k^{2}\left\langle\left\langle a_{\mathbf{k}}^{+}(0) a_{\mathbf{k}}(0)\right\rangle\left\langle a_{\mathbf{k}}(0) a_{\mathbf{k}}^{+}(0)\right\rangle\right\rangle E, \\
\left(\Phi, X_{1,2}\right) & =\frac{e \hbar}{m} \sum_{\mathbf{k}, \mathbf{k}^{\prime}}^{\prime} \mathbf{k k}^{\prime}\left\langle\left\langle a_{\mathbf{k}}^{+}(0) a_{\mathbf{k}^{\prime}}(0)\right\rangle\left\langle a_{\mathbf{k}^{\prime}}(0) a_{\mathbf{k}}^{+}(0)\right\rangle\right\rangle E .
\end{aligned}
$$

Каждый сомножитель первого слагаемого можно представить в первом порядке теории возмущений следующим образом:

$$
\left\langle a_{\mathbf{k}}^{+} a_{\mathbf{k}^{\prime}}\right\rangle=-\left\langle a_{\mathbf{k}^{\prime}} a_{\mathbf{k}}^{+}\right\rangle=w\left(\left|\mathbf{k}-\mathbf{k}^{\prime}\right|\right) \rho^{i}\left(\mathbf{k}^{\prime}-\mathbf{k}\right) \frac{n_{0}(k)-n_{0}\left(k^{\prime}\right)}{\varepsilon_{k}-\varepsilon_{k^{\prime}}} .
$$

Теперь во втором порядке теории возмущений

$$
\left(\Phi, X_{1,2}\right)=-\frac{N}{V^{2}} \frac{e \hbar}{m} \sum_{\mathbf{k}, \mathbf{k}^{\prime}} \mathbf{k k}^{\prime} w^{2}\left(\left|\mathbf{k}-\mathbf{k}^{\prime}\right|\right) S\left(\left|\mathbf{k}-\mathbf{k}^{\prime}\right|\right) \frac{\left[n_{0}(k)-n_{0}\left(k^{\prime}\right)\right]^{2}}{\left(\varepsilon_{k}-\varepsilon_{k^{\prime}}\right)^{2}} E .
$$

Далее мы учтем, что

$$
\begin{aligned}
& {\left[n_{0}(k)-n_{0}\left(k^{\prime}\right)\right]^{2}=} \\
& \quad=n_{0}(k)\left[1-n_{0}\left(k^{\prime}\right)\right]+n_{0}\left(k^{\prime}\right)\left[1-n_{0}(k)\right]-n_{0}(k)\left[1-n_{0}(k)\right]-n_{0}\left(k^{\prime}\right)\left[1-n_{0}\left(k^{\prime}\right)\right] .
\end{aligned}
$$

Объединяя $\left(\Phi, X_{1,2}\right)$ и $\left(\Phi, X_{2}\right)$, получим

$$
\begin{aligned}
\left(\Phi, X_{1,2}\right)+\left(\Phi, X_{2}\right)= & \frac{N}{V^{3} k_{\mathrm{B}} T} \frac{e \hbar}{m} \sum_{\mathbf{k}, \mathbf{k}^{\prime}}^{\prime} w^{2}\left(\left|\mathbf{k}-\mathbf{k}^{\prime}\right|\right) S\left(\left|\mathbf{k}-\mathbf{k}^{\prime}\right|\right) \times \\
& \times\left\{2 k^{2} \frac{n_{0}(\mathbf{k})\left[1-n_{0}(\mathbf{k})\right]}{\left(\varepsilon_{\mathbf{k}}-\varepsilon_{\mathbf{k}^{\prime}}\right)^{2}}-\left(k^{2}+k^{\prime 2}\right) \frac{n_{0}(\mathbf{k})\left[1-n_{0}\left(\mathbf{k}^{\prime}\right)\right]}{\left(\varepsilon_{\mathbf{k}}-\varepsilon_{\mathbf{k}^{\prime}}\right)^{2}}\right\} E .
\end{aligned}
$$


В более симметричном виде это выражение можно записать следующим образом:

$$
\begin{gathered}
\left(\Phi, X_{1,2}\right)+\left(\Phi, X_{2}\right)=\frac{N}{V^{3} k_{\mathrm{B}} T} \frac{e \hbar}{m} \sum_{\mathbf{k}, \mathbf{k}^{\prime}}^{\prime} \frac{k^{2}}{\left(\varepsilon_{\mathbf{k}}-\varepsilon_{\mathbf{k}^{\prime}}\right)^{2}} w^{2}\left(\left|\mathbf{k}-\mathbf{k}^{\prime}\right|\right) S\left(\left|\mathbf{k}-\mathbf{k}^{\prime}\right|\right) \times \\
\quad \times\left\{2 n_{0}(\mathbf{k})\left[1-n_{0}(\mathbf{k})\right]-n_{0}(\mathbf{k})\left[1-n_{0}\left(\mathbf{k}^{\prime}\right)\right]-n_{0}\left(\mathbf{k}^{\prime}\right)\left[1-n_{0}(\mathbf{k})\right]\right\} E .
\end{gathered}
$$

Последнее выражение существенно упрощается в случае сильно вырожденного или классического электронного газа. Первый случай реализуется для металлов, второй - для полностью ионизованной классической плазмы этих металлов. Для металлов $k_{\mathrm{B}} T \ll \varepsilon_{\mathrm{F}}$. В этом случае полученное выражение можно представить как

$$
\begin{aligned}
\frac{\left(\Phi, X_{1,2}\right)}{k_{\mathrm{B}} T}+\frac{\left(\Phi, X_{2}\right)}{k_{\mathrm{B}} T}= & \frac{N}{k_{\mathrm{B}} T V^{3}} \frac{e \hbar}{m} \sum_{\mathbf{k}, \mathbf{k}^{\prime}}^{\prime} \frac{k^{2}}{\left(\varepsilon_{\mathbf{k}}-\varepsilon_{\mathbf{k}^{\prime}}\right)^{2}} w^{2}\left(\left|\mathbf{k}-\mathbf{k}^{\prime}\right|\right) S\left(\left|\mathbf{k}-\mathbf{k}^{\prime}\right|\right) \times \\
& \times n_{0}(\mathbf{k})\left[1-n_{0}(\mathbf{k})\right]\left\{2-\frac{1-n_{0}\left(\mathbf{k}^{\prime}\right)}{1-n_{0}(\mathbf{k})}-\frac{n_{0}\left(\mathbf{k}^{\prime}\right)}{n_{0}(\mathbf{k})}\right\} E .
\end{aligned}
$$

Учитывая, что в предельном случае низких температур

$$
\frac{n_{0}(\mathbf{k})\left[1-n_{0}(\mathbf{k})\right]}{k_{\mathrm{B}} T}=\delta\left(\varepsilon_{k}-\varepsilon_{\mathrm{F}}\right)
$$

получим, что

$$
\begin{gathered}
\delta\left(\varepsilon_{k}-\varepsilon_{\mathrm{F}}\right) \frac{1}{1-n_{0}(\mathbf{k})}=\delta\left(\varepsilon_{k}-\varepsilon_{\mathrm{F}}\right) \frac{1}{n_{0}(\mathbf{k})}=2 \delta\left(\varepsilon_{k}-\varepsilon_{\mathrm{F}}\right), \\
\frac{\left(\Phi, X_{1,2}\right)}{k_{\mathrm{B}} T}+\frac{\left(\Phi, X_{2}\right)}{k_{\mathrm{B}} T}=0 .
\end{gathered}
$$

Таким образом, в предельном случае низких температур вклад перекрестных процессов рассеивания в электрическое сопротивление равен нулю. Естественно, что этот вклад будет отличным от нуля при учете температурных поправок.

Рассмотрим теперь классический электронный газ. Здесь можно пренебречь произведением функций распределения электронов проводимости по отношению к их первой степени. Тогда

$$
\left(\Phi, X_{1,2}\right)+\left(\Phi, X_{2}\right)=\frac{N}{V^{3}} \frac{4 e \hbar m}{\hbar^{3}} \sum_{\mathbf{k}, \mathbf{k}^{\prime}}^{\prime} \frac{n_{0}(k)}{\left(k+k^{\prime}\right)\left(k-k^{\prime}\right)} w^{2}\left(\left|\mathbf{k}-\mathbf{k}^{\prime}\right|\right) S\left(\left|\mathbf{k}-\mathbf{k}^{\prime}\right|\right) E .
$$

Придадим интегралу максимально простой вид для упрощения дальнейших численных расчетов. После перехода от суммирования к интегрированию в сферической системе координат нам нужно проинтегрировать лишь по двум модулям волновых векторов и углу между ними. В этом случае

$$
\begin{aligned}
\left(\Phi, X_{1,2}\right)+\left(\Phi, X_{2}\right)= & E \frac{N}{V} \frac{2 e \hbar m}{\pi^{4} \hbar^{3}} \int_{0}^{\infty} d k k n_{0}(k) \int_{0}^{\infty} d k^{\prime} \frac{k^{\prime}}{\left(k+k^{\prime}\right)\left(k-k^{\prime}\right)} \times \\
& \times \int_{\left|k-k^{\prime}\right|}^{\left|k+k^{\prime}\right|} w^{2}(q) S(q) q d q
\end{aligned}
$$




\section{7. ПЕРЕНОРМИРОВКА ЭНЕРГИИ СВОБОДНЫХ ЭЛЕКТРОНОВ}

В третьем уравнении цепочки для искомой функции Грина сохраним члены, соответствующие перенормировке энергии свободных электронов во втором уравнении цепочки, т. е. члены типа

$$
\frac{\left\langle\left\langle W(-\mathbf{q}) W\left(-\mathbf{q}^{\prime}\right) W\left(\mathbf{q}^{\prime}\right) a_{\mathbf{k}}^{+}(t) a_{\mathbf{k}+\mathbf{q}}(t) I_{z}(0)\right\rangle\right\rangle_{\omega}}{\varepsilon_{\mathbf{k}}-\varepsilon_{\mathbf{k}+\mathbf{q}+\mathbf{q}^{\prime}}+\hbar \omega},
$$

которые модифицируют второе уравнение исходной цепочки следующим образом:

$$
\begin{aligned}
\left(\varepsilon_{\mathbf{k}}-\right. & \left.\varepsilon_{\mathbf{k}+\mathbf{q}}+\hbar \omega\right)\left\langle\left\langle a_{\mathbf{k}}^{+}(t) \rho^{i}(-\mathbf{q}) a_{\mathbf{k}+\mathbf{q}}(t) \mathbf{I}(0)\right\rangle\right\rangle_{\omega}=\left\langle a_{\mathbf{k}}^{+}(0) \rho^{i}(-\mathbf{q}) a_{\mathbf{k}+\mathbf{q}}(0) \mathbf{I}(0)\right\rangle+ \\
& +V^{-1} w(q)\left\{\left\langle\left\langle a_{\mathbf{k}}^{+}(0) \rho(-\mathbf{q}) \rho(\mathbf{q}) a_{\mathbf{k}}(0) \mathbf{I}(0)\right\rangle\right\rangle_{\omega}-\right. \\
& \left.-\left\langle\left\langle a_{\mathbf{k}+\mathbf{q}}^{+}(t) \rho(\mathbf{q}) \rho(-\mathbf{q}) a_{\mathbf{k}+\mathbf{q}}(t) \mathbf{I}(0)\right\rangle\right\rangle_{\omega}\right\}+ \\
& +V^{-2} \sum_{\mathbf{q}^{\prime}}^{\prime} w^{2}\left(q^{\prime}\right)\left\{\frac{\left\langle\left\langle\rho(-\mathbf{q}) \rho\left(-\mathbf{q}^{\prime}\right) \rho\left(\mathbf{q}^{\prime}\right) a_{\mathbf{k}}^{+}(t) a_{\mathbf{k}+\mathbf{q}}(t) I_{z}(0)\right\rangle\right\rangle_{\omega}}{\varepsilon_{\mathbf{k}}-\varepsilon_{\mathbf{k}+\mathbf{q}+\mathbf{q}^{\prime}}+\hbar \omega}-\right. \\
& \left.-\frac{\left\langle\left\langle\rho(-\mathbf{q}) \rho\left(-\mathbf{q}^{\prime}\right) \rho\left(\mathbf{q}^{\prime}\right) a_{\mathbf{k}}^{+}(t) a_{\mathbf{k}+\mathbf{q}}(t) I_{z}(0)\right\rangle\right\rangle_{\omega}}{\varepsilon_{\mathbf{k}+\mathbf{q}^{\prime}}-\varepsilon_{\mathbf{k}+\mathbf{q}}+\hbar \omega}\right\}+ \\
& +V^{-1} \sum_{\mathbf{q}^{\prime}}^{\prime} w\left(q^{\prime}\right)\left\{\left\langle\left\langle a_{\mathbf{k}}^{+}(t) \rho(-\mathbf{q}) \rho\left(-\mathbf{q}^{\prime}\right) a_{\mathbf{k}+\mathbf{q}+\mathbf{q}^{\prime}}(t) \mathbf{I}(0)\right\rangle\right\rangle_{\omega}-\right. \\
& \left.-\left\langle\left\langle a_{\mathbf{k}+\mathbf{q}^{\prime}}^{+}(t) \rho(\mathbf{q}) \rho\left(-\mathbf{q}^{\prime}\right) a_{\mathbf{k}+\mathbf{q}}(t) \mathbf{I}(0)\right\rangle\right\rangle_{\omega}\right\} .
\end{aligned}
$$

Формально после расцепления функций Грина, содержащих слабо связанные средние, это уравнение можно записать в прежнем виде:

$$
\begin{aligned}
\left(\tilde{\varepsilon}_{\mathbf{k}}-\right. & \left.\tilde{\varepsilon}_{\mathbf{k}+\mathbf{q}}+\hbar \omega\right)\left\langle\left\langle a_{\mathbf{k}}^{+}(t) \rho^{i}(-\mathbf{q}) a_{\mathbf{k}+\mathbf{q}}(t) \mathbf{I}(0)\right\rangle\right\rangle_{\omega}=\left\langle a_{\mathbf{k}}^{+}(0) \rho^{i}(-\mathbf{q}) a_{\mathbf{k}+\mathbf{q}}(0) \mathbf{I}(0)\right\rangle+ \\
& +V^{-1} w(q)\left\{\left\langle\left\langle a_{\mathbf{k}}^{+}(t) \rho(-\mathbf{q}) \rho(\mathbf{q}) a_{\mathbf{k}}(t) \mathbf{I}(0)\right\rangle\right\rangle_{\omega}-\right. \\
& \left.-\left\langle\left\langle a_{\mathbf{k}+\mathbf{q}}^{+}(t) \rho(\mathbf{q}) \rho(-\mathbf{q}) a_{\mathbf{k}+\mathbf{q}}(t) \mathbf{I}(0)\right\rangle\right\rangle_{\omega}\right\}+ \\
& +V^{-1} \sum_{\mathbf{q}^{\prime}}^{\prime}\left\{w\left(\mathbf{q}^{\prime}\right)\left\langle\left\langle a_{\mathbf{k}}^{+}(t) \rho(-\mathbf{q}) \rho\left(-\mathbf{q}^{\prime}\right) a_{\mathbf{k}+\mathbf{q}+\mathbf{q}^{\prime}}(t) \mathbf{I}(0)\right\rangle\right\rangle_{\omega}-\right. \\
& \left.-\left\langle\left\langle a_{\mathbf{k}+\mathbf{q}^{\prime}}^{+}(t) \rho(\mathbf{q}) \rho\left(-\mathbf{q}^{\prime}\right) a_{\mathbf{k}+\mathbf{q}}(t) \mathbf{I}(0)\right\rangle\right\rangle_{\omega}\right\}
\end{aligned}
$$

где в статическом случае

$$
\begin{aligned}
\tilde{\varepsilon}_{\mathbf{k}} & =\varepsilon_{\mathbf{k}}-\frac{N}{V^{2}} \sum_{\mathbf{q}^{\prime}}^{\prime} \frac{w^{2}\left(q^{\prime}\right) S\left(q^{\prime}\right)}{\varepsilon_{\mathbf{k}}-\varepsilon_{\mathbf{k}+\mathbf{q}+\mathbf{q}^{\prime}}+i \delta}, \\
\tilde{\varepsilon}_{\mathbf{k}+\mathbf{q}} & =\varepsilon_{\mathbf{k}+\mathbf{q}}+\frac{N}{V^{2}} \sum_{\mathbf{q}^{\prime}}^{\prime} \frac{w^{2}\left(q^{\prime}\right) S\left(q^{\prime}\right)}{\varepsilon_{\mathbf{k}+\mathbf{q}^{\prime}}-\varepsilon_{\mathbf{k}+\mathbf{q}}+i \delta} .
\end{aligned}
$$

Теперь вклад второго порядка в вероятность перехода электронов между состояниями содержит перенормированные энергии, т. е. учитывает эффекты четвертого 
порядка:

$$
L_{2}\left(\mathbf{k}^{\prime}, \mathbf{k}\right)=3 \frac{2 \pi N}{V \hbar} \frac{w^{2}\left(\left|\mathbf{k}-\mathbf{k}^{\prime}\right|\right)}{\varepsilon^{2}\left(\left|\mathbf{k}-\mathbf{k}^{\prime}\right|\right)} S\left(\left|\mathbf{k}-\mathbf{k}^{\prime}\right|\right) \frac{1}{\pi} \frac{\sum^{\prime \prime}\left(k, k^{\prime}\right)}{\left[\varepsilon_{\mathbf{k}}-\varepsilon_{\mathbf{k}^{\prime}}+\sum^{\prime}\left(k, k^{\prime}\right)\right]^{2}+\left[\sum^{\prime \prime}\left(k, k^{\prime}\right)\right]^{2}},
$$

где

$$
\begin{aligned}
& \sum^{\prime}\left(k, k^{\prime}\right)=-\frac{N}{V^{2}} P \sum_{\mathbf{q}^{\prime}}^{\prime} w^{2}\left(q^{\prime}\right) S\left(q^{\prime}\right)\left\{\frac{1}{\varepsilon_{\mathbf{k}}-\varepsilon_{\mathbf{k}^{\prime}+\mathbf{q}^{\prime}}}+\frac{1}{\varepsilon_{\mathbf{k}+\mathbf{q}^{\prime}}-\varepsilon_{\mathbf{k}^{\prime}}}\right\} \\
& \sum^{\prime \prime}\left(k, k^{\prime}\right)=\pi \frac{N}{V^{2}} P \sum_{\mathbf{q}^{\prime}}^{\prime} w^{2}\left(q^{\prime}\right) S\left(q^{\prime}\right)\left\{\delta\left(\varepsilon_{\mathbf{k}}-\varepsilon_{\mathbf{k}^{\prime}+\mathbf{q}^{\prime}}\right)+\delta\left(\varepsilon_{\mathbf{k}+\mathbf{q}^{\prime}}-\varepsilon_{\mathbf{k}^{\prime}}\right)\right\} .
\end{aligned}
$$

Вклад третьего порядка в вероятность перехода электронов между состояниями также будет содержать перенормированные энергии свободных электронов, т. е. учитывать эффекты пятого порядка, и т. д.

\section{8. ЧЕТВЕРТЫЙ ПОРЯДОК ТЕОРИИ ВОЗМУЩЕНИЙ}

Учет членов четвертого порядка теории возмущений требует использования функции распределения взаимодействующего электронного газа. Не вдаваясь в детали ее вычисления, заметим, что при $T \rightarrow 0$ эта функция и в случае учета взаимодействия электронов с ионами имеет вид ступеньки. При этом

$$
\frac{n(k)[1-n(k)]}{k_{\mathrm{B}} T}=\delta\left[\varepsilon_{\mathbf{k}}+\sum^{\prime}(k)-\mu\right]
$$

где $\sum^{\prime}(k)$ - вещественная часть массового оператора электронов проводимости. Учтем этот эффект в кинетическом уравнении. Теперь оно будет иметь вид

$$
\frac{e \hbar}{m k_{\mathrm{B}} T} \mathbf{k} n(\mathbf{k})[1-n(\mathbf{k})] E=\frac{1}{k_{\mathrm{B}} T} \sum_{\mathbf{k}^{\prime}}\left[\Phi(\mathbf{k})-\Phi\left(\mathbf{k}^{\prime}\right)\right]^{2} n\left(\mathbf{k}^{\prime}\right)[1-n(\mathbf{k})] L\left(\mathbf{k}^{\prime}, \mathbf{k}\right) .
$$

Здесь мы опустили вклад перекрестных процессов, поскольку в четвертом порядке теории возмущений он оказался равным нулю. Теперь полевой вклад в коэффициент электрического сопротивления можно записать в виде

$$
\frac{\left(\Phi, X_{1,1}\right)}{k_{\mathrm{B}} T}=\frac{e \hbar}{m} \sum_{\mathbf{k}} k^{2} \delta\left[\varepsilon_{k}+\sum^{\prime}(k)-\mu\right] E .
$$

Вводя новую переменную - энергию электрона $\varepsilon=\varepsilon_{k}+\sum^{\prime}(k)-$ и переходя от суммирования по волновому вектору к интегрированию по энергии, получим

$$
\frac{\left(\Phi, X_{1,1}\right)}{k_{\mathrm{B}} T}=E \frac{e \hbar}{m} \int_{0}^{\infty} \delta(\varepsilon-\mu) k^{2}(\varepsilon) G(\varepsilon) d \varepsilon,
$$

где $G(\varepsilon)$ - плотность состояний взаимодействующего электронного газа. Учитывая, что при нулевой температуре $\mu=\varepsilon_{\mathrm{F}}$, имеем

$$
\frac{\left(\Phi, X_{1,1}\right)}{k_{\mathrm{B}} T}=E \frac{e \hbar}{m} k_{\mathrm{F}}^{2} G\left(\varepsilon_{\mathrm{F}}\right) .
$$


Вероятность переходов между состояниями теперь имеет вид

$$
\begin{aligned}
L\left(\mathbf{k}^{\prime}, \mathbf{k}\right)= & L_{2}\left(\mathbf{k}^{\prime}, \mathbf{k}\right)+L_{3}\left(\mathbf{k}^{\prime}, \mathbf{k}\right)+L_{4}\left(\mathbf{k}^{\prime}, \mathbf{k}\right) \\
L_{2}\left(\mathbf{k}^{\prime}, \mathbf{k}\right)= & 3 \frac{2 \pi N}{V \hbar} \frac{w^{2}\left(\left|\mathbf{k}-\mathbf{k}^{\prime}\right|\right)}{\varepsilon^{2}\left(\left|\mathbf{k}-\mathbf{k}^{\prime}\right|\right)} S\left(\left|\mathbf{k}-\mathbf{k}^{\prime}\right|\right) \times \\
& \times \frac{1}{\pi} \frac{\sum^{\prime \prime}\left(k, k^{\prime}\right)}{\left[\varepsilon_{k}-\varepsilon_{k^{\prime}}+\sum^{\prime}\left(k, k^{\prime}\right)\right]^{2}+\left[\sum^{\prime \prime}\left(k, k^{\prime}\right)\right]^{2}},
\end{aligned}
$$

где для простоты мы выписали явно только перенормированный член второго порядка. Заметим, что при учете эффектов не старше четвертого порядка перенормировку энергий достаточно учитывать лишь в этом члене. Соответственно

$$
\frac{1}{k_{\mathrm{B}} T}(\Phi, \hat{L} \Phi)=\frac{1}{2 k_{\mathrm{B}} T} \sum_{\mathbf{k}, \mathbf{k}^{\prime}}\left(k^{2}+k^{\prime 2}-2 \mathbf{k k}^{\prime}\right) n\left(\mathbf{k}^{\prime}\right)[1-n(\mathbf{k})] L\left(\mathbf{k}^{\prime}, \mathbf{k}\right) .
$$

Учитывая, что $L\left(\mathbf{k}^{\prime}, \mathbf{k}\right) \propto \delta\left(\varepsilon_{\mathbf{k}}-\varepsilon_{\mathbf{k}^{\prime}}\right)$, последнее выражение можно записать как

$$
\frac{1}{k_{\mathrm{B}} T}(\Phi, \hat{L} \Phi)=\sum_{\mathbf{k}} \frac{n(\mathbf{k})[1-n(\mathbf{k})]}{k_{\mathrm{B}} T} k^{2} \sum_{\mathbf{k}^{\prime}}\left[1-\cos \left(\widehat{\mathbf{k k}^{\prime}}\right)\right] L\left(\mathbf{k}^{\prime}, \mathbf{k}\right) .
$$

Заметим, что само понятие времени релаксации имеет смысл только при выполнении последнего соотношения. Сделанное утверждение относительно вероятности перехода является удобным, но не необходимым. Без этого предположения нам следует отказаться от удобного термина - времени релаксации, но схема расчета при этом не меняется.

Переходя в первой сумме к интегрированию по энергии, получим

$$
\frac{1}{k_{\mathrm{B}} T}(\Phi, \hat{L} \Phi)=k_{\mathrm{F}}^{2} G\left(\varepsilon_{\mathrm{F}}\right) \sum_{\mathbf{k}^{\prime}}\left[1-\cos \left(\widehat{\mathbf{k k}^{\prime}}\right)\right] L\left(\mathbf{k}^{\prime}, \mathbf{k}\right) .
$$

Теперь выражение для коэффициента электрического сопротивления будет иметь вид

$$
\rho=\frac{m}{e^{2} n_{e}} \frac{G_{0}\left(\varepsilon_{\mathrm{F}}\right)}{G\left(\varepsilon_{\mathrm{F}}\right)} \frac{1}{\tau\left(\varepsilon_{\mathrm{F}}\right)},
$$

где мы ввели плотность состояний свободных электронов

$$
G_{0}\left(\varepsilon_{\mathrm{F}}\right)=V \frac{m}{\pi^{2} \hbar^{2}} k_{\mathrm{F}},
$$

обратное время релаксации равно

$$
\frac{1}{\tau\left(\varepsilon_{\mathrm{F}}\right)}=\frac{1}{3 V} \sum_{\mathbf{k}^{\prime}} L\left(\mathbf{k}^{\prime}, \mathbf{k}\right)\left[1-\cos \left(\widehat{\mathbf{k k}^{\prime}}\right)\right]
$$

a $n_{e}=k_{\mathrm{F}}^{3} / 3 \pi^{2}$. Входящий сюда волновой вектор Ферми следует вычислять для взаимодействующего электронного газа.

Общее выражение для вклада четвертого порядка в обратное время релаксации было получено еще в работах [13], [14]. Оно не имеет практической пользы из-за своей громоздкости, поэтому здесь мы его не приводим. Актуальной остается задача приближенного суммирования всего ряда теории возмущений для обратного времени релаксации. 


\section{9. ОБСУЖДЕНИЕ И ВЫВОДЫ}

На основе вариационного принципа нами получено выражение для коэффициента электрического сопротивления простых неупорядоченных металлов, справедливое не ниже четвертого порядка теории возмущений по электрон-ионному взаимодействию. Ионная подсистема при этом считается статической, а температурные поправки не учитываются.

Коэффициент электропроводности определяется не только временем релаксации, но и плотностью состояний электронов проводимости. Этот факт чрезвычайно важен для объяснения экспериментально наблюдаемых отклонений постоянной Холла жидких металлов от постоянной Холла идеального электронного газа. Существенную роль зависимость коэффициента электрического сопротивления от плотности состояний играет и при объяснении поведения сопротивления неупорядоченных металлов вблизи перехода металл-диэлектрик.

Оказалось, что перекрестные процессы рассеяния электронов проводимости в пределе низких температур в четвертом порядке теории возмущений не дают вклада в электрическое сопротивление простых неупорядоченных металлов в отличие от случая полностью ионизованной плазмы. При отличных от нуля температурах они приводят к нарушению закона Видемана-Франца.

Параметры расцепления функций Грина старших порядков, возникающие при получении квантового кинетического уравнения, выбраны из условия полного совпадения уравнения Больцмана и квантового кинетического уравнения в низшем порядке теории возмущений. Это позволяет придать ясный физический смысл процедуре расцепления, состоящей в выполнении уравнения детального баланса, и максимально сблизить структуры этих уравнений при учете членов высших порядков теории возмущений в квантовом кинетическом уравнении.

\section{Список литературы}

[1] J. M. A. Ziman, Phil. Mag., 6:68 (1961), 1013-1034.

[2] В. В. Немошкаленко, А. В. Романова, А. Г. Ильинский и др., Аморфные металлические сплавы, Наукова думка, Киев, 1987.

[3] В. Т. Швец, Метод функиий Грина в теории металлов, Латстар, Одесса, 2002.

[4] B. Springer, Phys. Rev., 136:1A (1964), 115-124.

[5] B. Springer, Phys. Rev., 154:3 (1967), 614-621.

[6] T. Neal, Phys. Rev., 169:3 (1968), 508-516.

[7] T. Neal, Phys. Fluids, 13:2 (1970), 249-262.

[8] N. W. Ashcroft, W. Schaich, Phys. Rev. B, 1:4 (1970), 1370-1379; Erratum, 3:4 (1971), 1511.

[9] N. W. Ashcroft, W. Schaich, Phys. Rev. B, 3:4 (1971), 1511-1511.

[10] A. Bringer, D. Wagner, Z. Phys., 241:4 (1971), 295-307.

[11] J. Popielawski, Physica, 78:1 (1974), 97-112.

[12] J. Gorecki, J. Popielawski, J. Phys. F, 13:10 (1983), 2107-2114.

[13] В. Т. Швец, ТМФ, 86:1 (1991), 111-119.

[14] V. T. Shvets, Mat. Sci. and Engrg. B, 26:2-3 (1994), 141-145.

[15] В. Т. Швец, Э. В. Белов, А. В. Кущак, Металлофиз. новейшие технол., 20:6 (1998), $3-14$.

[16] В. Т. Швец, Э.В.Белов, УФЖ, 44:11 (1999), 1408-1413. 
[17] V. T. Shvets, E. V. Belov, Acta Phys. Polonica A, 96:6 (1999), 741-750.

[18] В. Т. Швец, Металлобиз. новейшие технол., 23:6 (2001), 745-753.

[19] V. Shvets, S. Savenko, S. Datsko, Condensed Matter Phys., 5:3(31) (2002), 511-522.

[20] В. Т. Швец, ФМM, 103:4 (2007), 346-352.

[21] В. Т. Швец, ЖЭТФ, 131:4 (2007), 743-749.

[22] В. Т. Швец, А. Г. Власенко, А. Д. Буханенко, Писъма в ЖЭТФ, 86:8 (2007), 625-629.

[23] Д. Н. Зубарев, В. Г. Морозов, Г. Рёпке, Статистическая механика неравновесных процессов, т. 2, Физматлит, М., 2002.

[24] Н. М. Плакида, ЖЭТФ, 53:12 (1967), 2041-2052.

[25] С. В. Тябликов, Методы квантовой теории магнетизма, Наука, М., 1975.

[26] Дж. Займан, Электроны и фононы. Теория явлений переноса в твердых телах, ИЛ, M., 1962.

Поступила в редакцию 19.06.2010, после доработки 11.09.2010 